\title{
Características de um Professor Exemplar: Percepções de Estudantes e Professores
}

\section{The Characteristics of a Model Teacher from the Viewpoint of Students and Teachers}

Lara Gurgel Fernandes Távora ${ }^{I}$ iD Laila Teixeira Amorim ${ }^{I}$ (D) João Pedro Brasil Teixeira ${ }^{I}$ (D

Heitor Moita Mota ${ }^{I}($ D

Thais Barroso Vieira Costa ${ }^{I}$ (iD

Victor Fernandes Távora Vieira Costa ${ }^{I}$ (iD

\section{RESUMO}

Introdução: Na área médica, a forma de ensinar e aprender vem mudando com o tempo. Dessa forma, a investigação dos atributos que tornam um professor exemplar é importante para formular novas estratégias de ensino na educação médica. O presente estudo objetiva avaliar a percepção dos discentes e docentes do curso de Medicina sobre quais qualidades o docente deve ter para ser considerado um modelo. Método: Trata de um estudo quantitativo, transversal, com estudantes e professores do curso de Medicina da Universidade de Fortaleza (Unifor). Foi aplicado aos participantes um questionário elaborado pelos autores sobre os atributos mais importantes para ser considerado um bom professor. Cada atributo deveria receber peso de 1 a 5 caso o participante o julgasse menos ou mais importante. Resultados: Participaram do estudo 74 professores e 344 alunos. As maiores médias nos dois grupos foram para a "atitude no ambiente de ensino" e "interação com os alunos e colegas", embora os professores tenham valorizado mais esses domínios ( $p=0,03$ e $p=0,006$, respectivamente). O domínio "características pessoais" foi menos valorizado pelos alunos $(p=0,02)$. No domínio "atitude no ambiente de ensino", facilitar a aprendizagem e ter uma atitude ética com o paciente foram as características mais valorizadas, embora os professores tenham valorizado ainda mais este último ( $p=$ 0,001). No domínio "interação com os alunos e colegas", a capacidade de estimular a participação do aluno foi considerada menos importante pelos alunos $(p=0,001)$. No domínio "características pessoais", ter entusiasmo pelo ensino foi bastante valorizado, entretanto os professores tenderam a dar um peso maior que os alunos ( $p$ = 0,001). A avaliação do domínio "desenvolvimento profissional" mostrou que o envolvimento com pesquisa foi o menos valorizado pelos participantes. Conclusões: Esses resultados sugerem que, para a identificação de um professor exemplar, os dois grupos valorizaram não apenas as atitudes relacionadas à docência, mas sobretudo as atitudes no ambiente de ensino e na interação com alunos e colegas. Sugerem ainda a necessidade de desenvolver estratégias de valorização da pesquisa científica, inclusive com incentivos para uma maior participação dos docentes e discentes, ressaltando a sua a importância na formação médica. 


\section{KEYWORDS}

- Medical Education.

- Medical Teacher.

- Medical Students.

\section{ABSTRACT}

Introduction: Learning and teaching methods have changed over time in medical education. Therefore, there is a strong case for investigating the attributes of a model professor in order to formulate new teaching strategies in medical education. This study aimed to ascertain which character traits are viewed in the eyes of medical students and teachers as essential to the profile of a model teacher. Methods: This is a quantitative, crosssectional study conducted with medical students and teachers of the University of Fortaleza (UNIFOR) School of Medicine. Participants were invited to respond to a questionnaire, developed by the authors, addressing the most important characteristics of a model teacher. The respondents were asked to score each characteristic from 1 to 5 to reflect its respective importance. Results: A total of 418 people were surveyed, of whom 74 were teachers and 344 students. The highest scoring characteristics in both groups were "Attitude in the teaching environment" and "Interaction with students and colleagues", although teachers placed more value on these attributes ( $p=$ 0.03 and $p=0.006$ respectively). "Personality traits" was the least appreciated by the students $(p=0.02)$. In the "Attitude in the teaching environment" category, facilitating learning and maintaining an ethical attitude with the patient were the most valued characteristics in both groups, although the latter was more appreciated by the teachers $(p=0.001)$. In the "Interaction with students and colleagues" category, the ability to stimulate student participation was considered least important by the students $(p=0.001)$. Enthusiasm for teaching scored highly in both groups in the "Personality traits" category, though teachers tended to give rate it more highly than the students $(p=0.001)$. The analysis of the "Professional development" category showed that, for both groups, involvement in research was the least valued characteristic. Conclusions: These results suggest that not only attributes associated with teaching practice, but also teachers' attitudes in the teaching environment and their interaction with students and colleagues were considered by both groups as important to their identification of a model teacher. They also suggest the necessity for developing strategies to value scientific research and stimulate greater participation of both students and teachers, underlining its importance in medical training.

Recebido em 11/4/20

Aceito em 23/4/20

\section{INTRODUÇÃO}

A formação do estudante de Medicina passa por muitas variáveis, como a relação entre aluno e professor. Dessa forma, o professor não é somente uma pessoa com mais conhecimento, mas também um modelo a ser seguido, o que é crucial na formação dos futuros médicos ${ }^{1}$. É necessário então identificar o perfil dos professores da área médica por meio da percepção dos alunos e dos próprios professores para saber que modelo de profissional idealizamos.

Irby e Papadakis ${ }^{2}$ identificaram seis características que estão associadas pelos alunos a um bom professor: conhecimento, organização, entusiasmo, habilidade de interação com o grupo, competência clínica e profissionalismo. Essas características aparecem em maior ou menor frequência, dependendo do nível do estudante que se está entrevistando. Já na visão do professor, algumas características que não são normalmente pontuadas pelos estudantes assumem um papel de relevância, como estar familiarizado com princípios de andragogia ${ }^{3}$.

A discussão em torno do conceito do que faz um bom professor na área médica vem mudando com o tempo, assim como a forma de ensinar e aprender. De acordo com Sutkin, Wagner, Harris e Schiffer ${ }^{4}$, a transformação do perfil dos estudantes requer novas formas de ensinar, e, nesse processo, os professores da área médica não devem apenas ser supervisores e transmissores de conhecimento, mas também exemplos a serem seguidos de valores e atributos da vida profissional. Para Haider, Snead e Bari ${ }^{5}$, por meio do conhecimento desses atributos, é possível traçar novas estratégias de ensino na área da educação médica, bem como reforçar essas qualidades na instituição de ensino. Dessa forma, a investigação dessa questão é extremamente importante para muitas respostas em relação à educação médica e para as instituições que são responsáveis pela formação de futuros médicos.

O presente estudo tem o objetivo de comparar a percepção de professores e estudantes de Medicina sobre quais características um professor exemplar deve ter.

\section{METODOLOGIA}

Trata-se de um estudo com abordagem quantitativa, transversal, realizado de julho a dezembro de 2018 com discentes e docentes do curso de Medicina da Universidade de Fortaleza (Unifor). Todos os professores e alunos do curso receberam o convite on-line para participação no estudo. Ao aceitarem participar, solicitou-se a todos que respondessem a um questionário também on-line contendo perguntas sobre quais características julgavam mais importantes para que um professor fosse considerado um exemplo. Esse questionário foi elaborado pelos autores especificamente para esse fim. Escolheram-se características consideradas importantes com base em alguns dados de literatura ${ }^{2-4}$. Essas características foram organizadas em domínios: "atitude no ambiente de ensino" (facilita o aprendizado, apresenta o plano de ensino, é organizado nas atividades, fornece feedback e mantém uma atitude ética com o paciente), "interação com os alunos e colegas" (mantém bom relacionamento com os alunos, tem boa comunicação e boa habilidade de interação com o grupo, apresenta empatia, apresenta-se disponível para ajudar, estimula a participação do aluno e estimula o raciocínio clínico), "características pessoais" (discreto, cordial, entusiasta pelo ensino, pontual, proativo, humilde, apto a receber 
críticas, habilidade de liderança e bom ouvinte) e "desenvolvimento profissional" (estar atualizado no que se propõe a ensinar, ser envolvido com pesquisa, ter competência clínica e utiliza novas formas de ensino). Os participantes avaliaram cada característica atribuindo pesos de 1 (considerada de menor importância) a 5 (considerada de maior importância) para cada uma delas.

$\mathrm{Na}$ análise, utilizou-se o programa SPSS. Calcularam-se medidas de frequência e tendência central. Para a comparação entre os pesos atribuídos pelos professores e alunos, foi utilizado teste Mann-Whitney, visto que todas as variáveis apresentaram uma distribuição diferente da normal. Considerou-se um $p$ significante $<0,05$.

A pesquisa foi aprovada pelo Comitê de Ética em Pesquisa da Unifor (Parecer $\mathrm{n}^{\circ}$ 2.606.341).

\section{RESULTADOS}

Foram incluídos um total de 418 participantes, sendo 74 professores e 344 alunos. A maioria dos participantes era do sexo feminino, tanto entre os professores quanto entre os alunos (66\% e 62\%, respectivamente). A distribuição dos participantes por categoria de semestre mostra uma predominância de professores com maior atuação em semestres clínicos (do quinto ao $12^{\circ}$ semestre) (64,9\%). Entre os alunos, entretanto, observouse uma distribuição mais equilibrada $(49,4 \%$ de semestres básicos (do primeiro ao quarto semestre) e 50,6\% de semestres clínicos).

A avaliação dos domínios mostrou que as maiores médias nos dois grupos foram para a "atitude no ambiente de ensino" e "interação com os alunos e colegas". Entretanto, em média, os professores tenderam a valorizar mais os domínios "atitude no ambiente de ensino", "interação com os alunos e colegas" e "características pessoais" quando comparados com os alunos. Este último, inclusive, foi o domínio menos valorizado pelos alunos (Gráfico 1).
Na avaliação do domínio "atitude no ambiente de ensino", "facilitação do aprendizado", "organizado nas atividades" e "mantém atitude ética com o paciente" foram as características consideradas mais importantes pelos dois grupos. Entretanto, observa-se que os docentes atribuíram, em média, pesos significativamente maiores para a adoção de uma atitude ética para com o paciente. "Apresentar o plano de ensino" obteve a menor média nas duas categorias, embora os professores ainda tenham valorizado mais essa atitude do que os alunos. Observa-se ainda uma valorização significativamente maior da realização do feedback pelos professores (Gráfico 2).

A avaliação do domínio "interação com os alunos e colegas" mostrou, em geral, uma média elevada na percepção dos dois grupos, com exceção da capacidade de estimular a participação do aluno, a qual foi considerada significativamente menos importante pelos alunos (Gráfico 3).

A análise do domínio "características pessoais" mostrou que ser discreto foi considerado menos importante para os dois grupos, embora a importância dada a essa característica pelos alunos tenha sido significativamente menor do que pelos professores. Outra diferença encontrada foi na importância dada ao fato de ser cordial e ter entusiasmo pelo ensino. Na percepção dos professores, essas características têm uma importância maior na formação do professor exemplar (Gráfico 4).

Apesar de, em média, não termos observado diferença entre os dois grupos quando avaliamos o domínio "desenvolvimento profissional", a sua análise detalhada mostrou que o fato de o professor utilizar novas formas de ensino foi mais valorizado pelos professores do que pelos alunos. Ter competência clínica e estar atualizado no que se propõe a ensinar foram as características mais valorizadas. Na percepção dos dois grupos, o envolvimento com pesquisa teve um peso menor para considerar um professor exemplar (Gráfico 5).

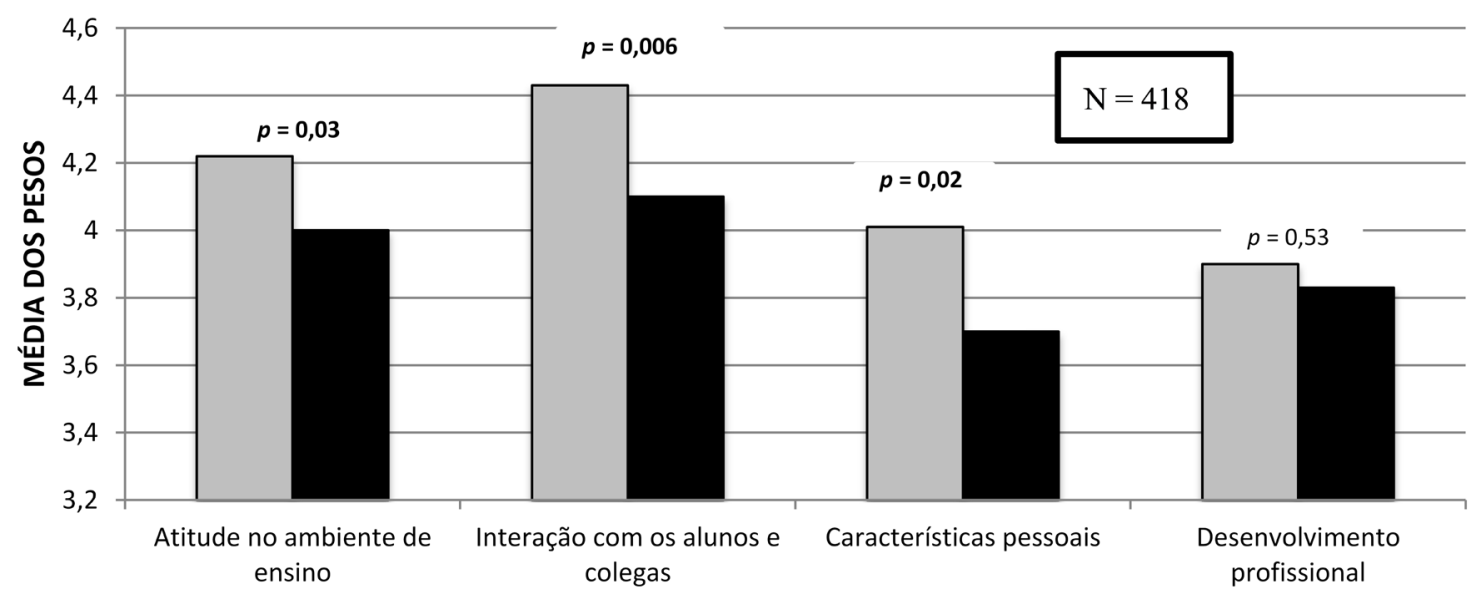

$\square$ PROFESSOR $\square$ ALUNO

Fonte: Elaborado pelos autores. 


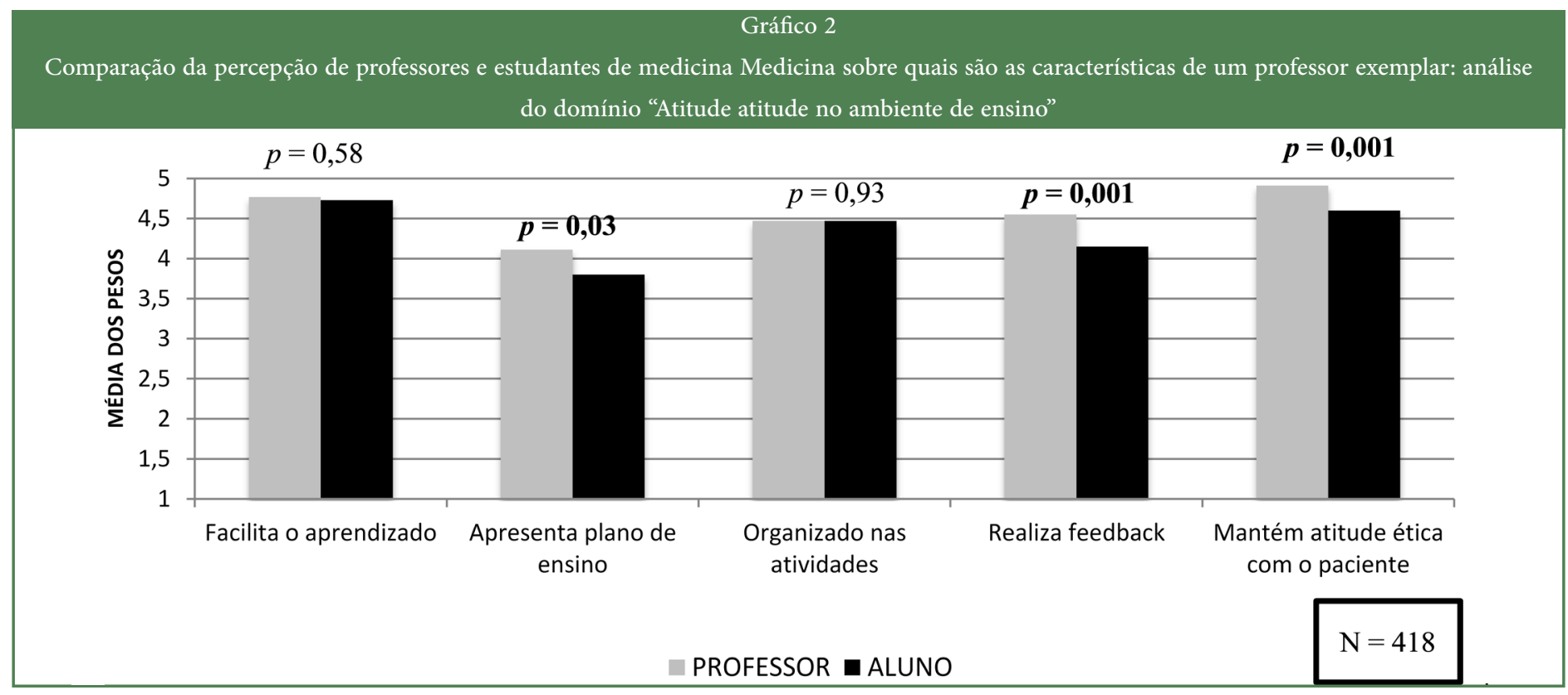

Fonte: Elaborado pelos autores.

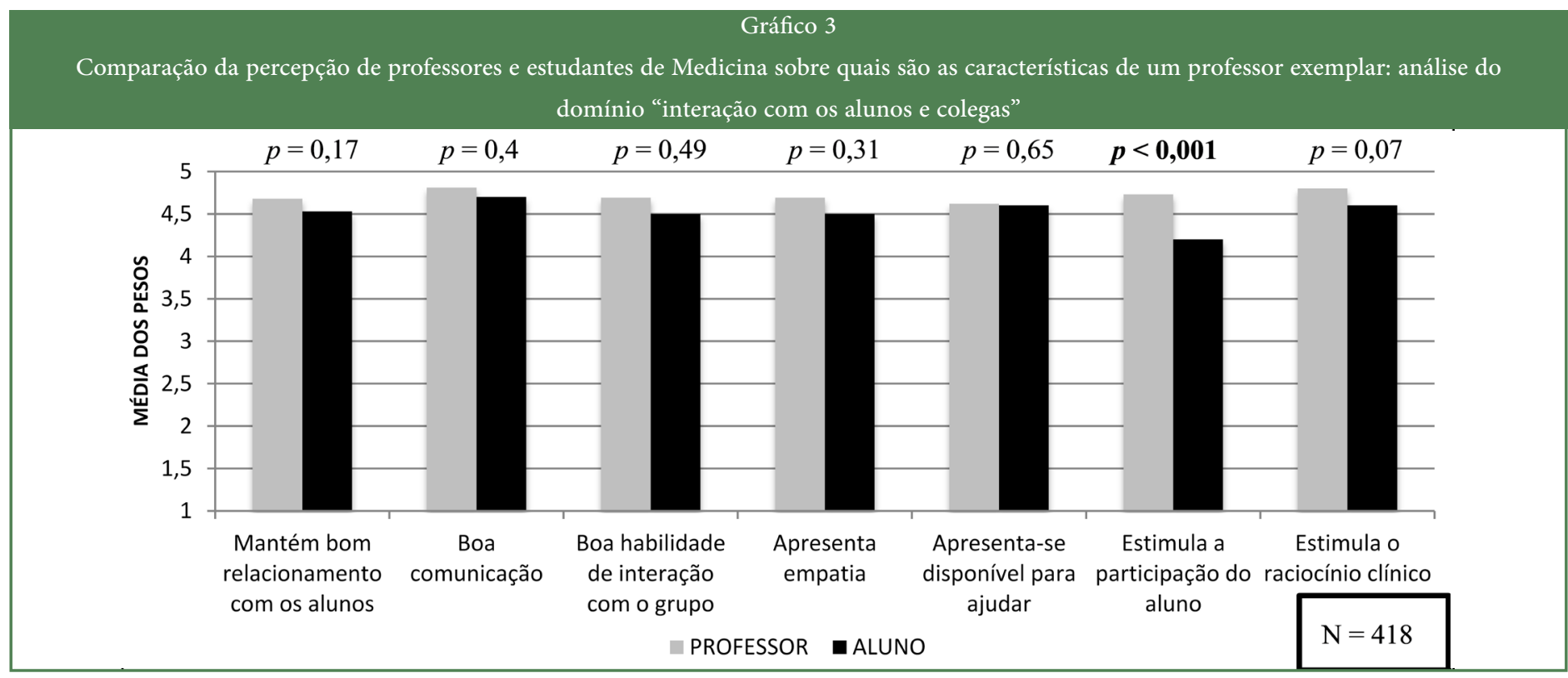

Fonte: Elaborado pelos autores.

\section{DISCUSSÃO}

$\mathrm{Na}$ avaliação do domínio "atitude no ambiente de ensino", "facilitar o aprendizado", "manter atitude ética com o paciente" e "ser organizado nas atividades" foram as atitudes mais valorizadas pelos participantes. Muitas dessas atitudes também foram apontadas como importantes em outros estudos $^{1}$. Em um estudo que avaliou a percepção de estudantes de Medicina sobre as qualidades essenciais de um professor, as características pessoais capacidade de liderança, organização nas atividades, inteligência, capacidade de se expressar e estímulo do raciocínio ético foram bastante valorizadas $^{6}$. A mudança de paradigma do professor tradicional, transmissor do conhecimento, para aquele que, sendo um modelo para os seus estudantes, assume o papel de facilitador da aquisição de conhecimentos transforma essa capacidade numa peça fundamental no processo de aprendizagem ${ }^{7}$.

Entende-se ainda que a influência do professor como exemplo acaba por ter impacto nas atitudes, nos comportamentos e, inclusive, nos valores éticos dos estudantes ${ }^{8}$. Nesse contexto, a valorização de uma atitude ética, tanto pelos docentes quanto pelos discentes, observada neste estudo, demonstra a importância que ela tem na construção de modelos de profissionais para os dois grupos. Apesar disso, observou-se uma valorização significativamente menor dessa atitude pelos estudantes. Talvez isso esteja relacionado com a necessidade de uma outra mudança de paradigma, desta vez na elaboração dos currículos de competência dos cursos de Medicina. 
Em estudo desenvolvido em uma universidade brasileira do Nordeste, foi observado que os estudantes de Medicina, durante a graduação, são treinados essencialmente para adquirir as habilidades técnicas necessárias, mas não para o desenvolvimento ético e moral. Esse estudo conclui que houve uma diminuição da competência moral quando se compararam os alunos dos ciclos básicos com aqueles dos ciclos clínicos. Esses dados podem justificar, em parte, a menor média encontrada nos pesos atribuídos à atitude ética evidenciados na nossa amostra ${ }^{9}$. Ademais, esses achados reforçam a necessidade de manter a discussão sobre ética médica como uma constante nas universidades, de modo a abordar não somente a ética médica, mas também a ética no ambiente da academia ${ }^{10,11}$.

Comparação da percepção de professores e estudantes de Medicina sobre quais são as características de um professor exemplar: análise do domínio "características pessoais"

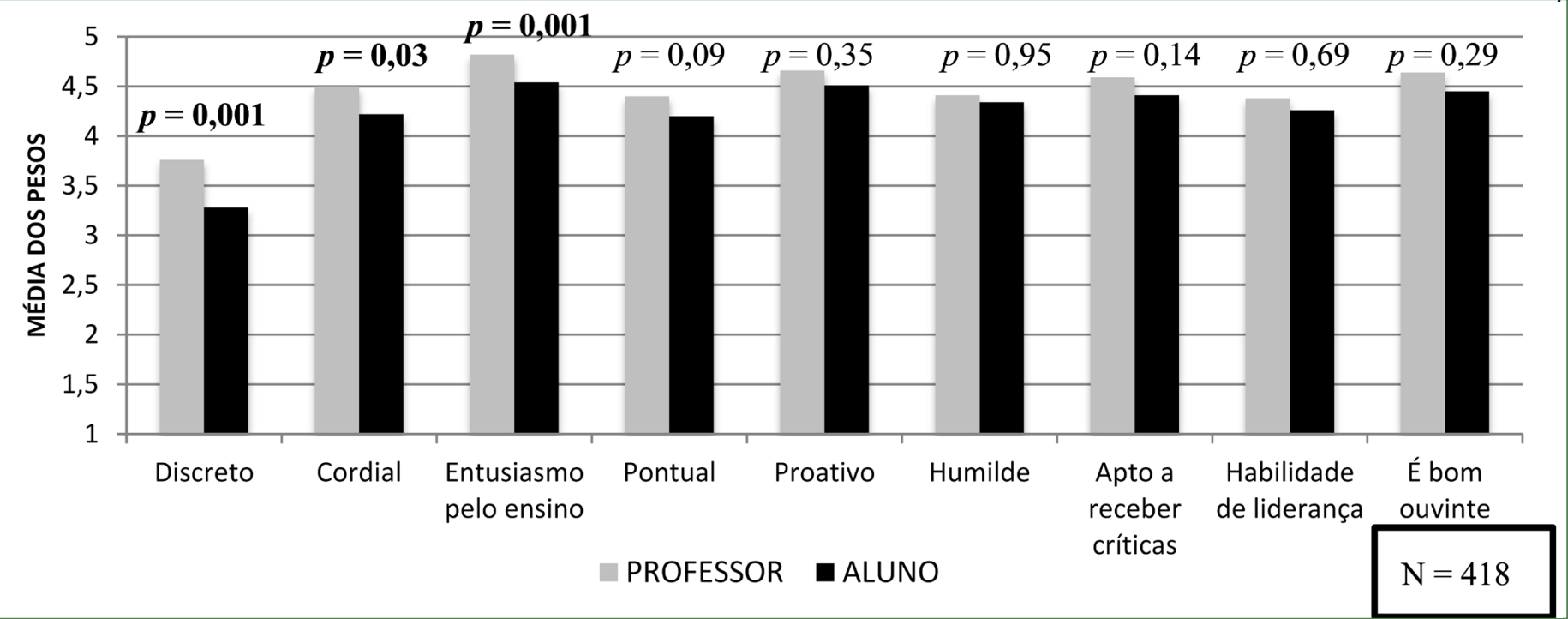

Fonte: Elaborado pelos autores.

\section{Gráfico 5}

Comparação da percepção de professores e estudantes de Medicina sobre quais são as características de um professor exemplar: análise do domínio "desenvolvimento profissional"

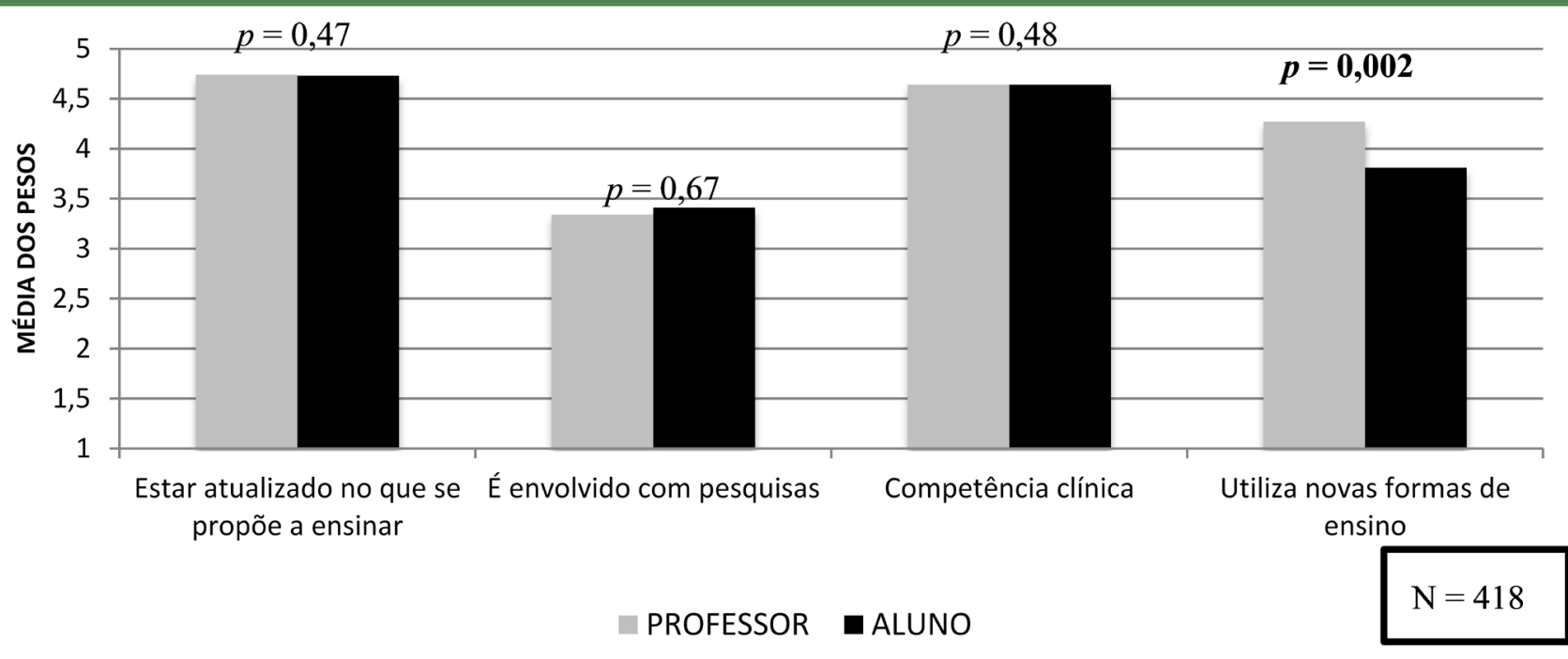

Fonte: Elaborado pelos autores. 
As atitudes "apresentar o plano de ensino" e "realizar feedback" foram menos valorizadas pelos alunos, apesar de serem direcionadas para o desenvolvimento profissional deles. No curso de Medicina da Unifor, essas atividades docentes fazem parte da programação dos planos de ensino, garantindo em cada módulo momentos protegidos para a apresentação do plano de ensino e realização de feedback. Dessa forma, na percepção dos estudantes, essas atividades deveriam ser intrínsecas à docência e não destinadas exclusivamente a tornar um professor diferenciado. Apesar desse achado, outros autores demonstraram a valorização da realização do feedback como uma atitude importante desempenhada pelo professor. Segundo Lewis, Patel e Lopreiato ${ }^{12}$, o feedback pode constituir-se em uma ferramenta poderosa de aprendizagem caso seja bem-feito e valorizado tanto pelos docentes quanto pelos discentes. Em um estudo realizado na Holanda, a capacidade de realizar um bom feedback foi apontada como uma competência essencial do professor, juntamente com as capacidades de comunicar-se bem e ter respeito pelos estudantes ${ }^{13}$.

$\mathrm{Na}$ avaliação do domínio "interação com alunos e colegas", todas as características foram consideradas importantes pelos participantes. Com efeito, observa-se que esse resultado se repete em outros estudos. A capacidade de interagir com os alunos é apontada pelos estudantes como uma habilidade essencial e engloba, em si, várias características importantes na relação de ensino e aprendizagem ${ }^{6,14}$. Vale ressaltar que, no presente estudo, a categoria "estimula a participação do aluno", entretanto, foi avaliada consideravelmente menos importante pelos alunos. Essa diferença pode ser explicada pelo ambiente de respeito mútuo entre professores e alunos existente dentro da própria universidade. Portanto, os estudantes já são incentivados a formular e argumentar suas opiniões sobre os diversos assuntos discutidos durante o curso, não considerando, então, o estímulo à participação por parte do professor como algo que o torne exemplar 5 .

Com exceção da característica de "ser discreto", todo o restante do domínio "características pessoais" foi bastante valorizado pelos dois grupos. Em relatório do Royal College of Physicians, o qual discute o avanço no profissionalismo médico, entre os sete aspectos considerados mais valorizados, é possível identificar dois que estão inseridos nesse domínio, quais sejam: capacidade de liderança e ser proativo/inovador ${ }^{15}$. Observa-se assim que a valorização das características desse domínio acaba por extrapolar os limites da comunidade universitária, sendo considerada por entidades médicas como essencial para a formação do profissional médico moderno. Nesse domínio, ser proativo e ter entusiasmo pelo ensino foram as características mais valorizadas, sendo esta última significativamente mais valorizada pelos professores. Essa importância dada pelos professores ao "entusiasmo pelo ensino" é similar ao que foi encontrado em estudos anteriores, que consideram essa uma característica bastante importante para um ensino efetivo ${ }^{16,17}$. Com o aparecimento de diversas novas formas de aprendizado e com a facilitação do acesso à informação, a relevância do entusiasmo do professor pelo ensino pode ter diminuído para os estudantes. O uso da tecnologia nas metodologias ativas é cada vez mais comum. Fazer transmissões de dados em alta velocidade e trocar informações em tempo real já é uma realidade na rotina de vários cursos de ensino superior. Dessa forma, o entusiasmo do professor pelo ensino pode dar lugar ao fascínio do acesso à informação que a tecnologia proporciona. Esse processo de renovação levanta a questão sobre a necessidade de transformação de metodologias pedagógicas e uma nova postura do docente ${ }^{18,19}$.

$\mathrm{Na}$ análise mais detalhada do domínio "desenvolvimento profissional", os professores valorizaram mais a utilização de novas metodologias de ensino quando comparados com os alunos. O curso de Medicina da Unifor tem um currículo integrado, cooperativo e baseado em metodologias de ensino-aprendizagem centradas no aluno. Dessa forma, práticas de problem based learning ( $\mathrm{PBL}$ ) com grupos tutorias e team based learning (TBL), sessões interativas e aula invertida são uma constante nos projetos de ensino ao longo dos semestres. Assim, acreditamos que, na percepção dos estudantes, essa característica não tornaria um professor exemplar, pois trata-se, na verdade, de metodologias de ensino utilizadas por todo e qualquer docente desse curso.

A observação de que tanto alunos quanto professores valorizaram pouco o envolvimento com pesquisa merece uma análise mais aprofundada. Zemlo, Garrison, Partridge e Ley ${ }^{20}$ observaram uma redução significativa do número de médicos pesquisadores e classificaram esse resultado de preocupante. Em um outro estudo, Ávila e Restrepo ${ }^{21}$ chegaram à mesma conclusão e questionam se o estudante deve simplesmente "consumir informação médica" ou "criar" ele mesmo essa informação. Os autores discutiram ainda sobre o impacto positivo que o envolvimento em pesquisa pode ter na prática clínica dos estudantes de Medicina. Levar para a comunidade médica a percepção da importância do envolvimento em pesquisa para a sua formação parece ser algo fundamental e necessário pelo que percebemos. São inúmeros os benefícios do envolvimento com pesquisa: desenvolvimento da capacidade de pensamento analítico, leitura crítica, habilidades de escrita, organização, disciplina, responsabilidade e comunicação em público. As competências desenvolvidas no aprendizado e envolvimento com pesquisas, sem dúvida, capacitam de forma diferenciada os profissionais médicos ${ }^{22}$. Em um estudo desenvolvido na Universidade Estadual do Ceará, Souza, Nunes, Silveira e Nobrega-Therrien ${ }^{23}$ concluíram que a participação em pesquisa foi maior entre os docentes não médicos que lecionavam no ciclo básico e tinham dedicação exclusiva. Sugeriram ainda que a elevada proporção de docentes médicos com vinculação de apenas 20 horas representava um fator que diminuía o seu envolvimento em pesquisa. Esses fatos podem justificar a menor valorização dada ao envolvimento em pesquisa por professores e estudantes.

\section{CONCLUSÃO E CONSIDERAÇÕES FINAIS}

Conclui-se, portanto, que as características que fazem de um professor exemplar apresentaram vários pontos em comum entre os dois grupos. Os domínios mais valorizados foram os relacionados à "interação com os alunos e colegas" e "atitude no ambiente de ensino". O domínio das "características pessoais" também foi bastante valorizado pelos participantes, com exceção da característica de "ser discreto". As características "apresentar plano de ensino" e "utilizar novas metodologias de ensino", que estão mais relacionadas ao exercício da docência, foram menos valorizadas, especialmente pelos discentes. Os dados mostraram também uma menor valorização do envolvimento em pesquisa pelos dois grupos.

Esses resultados sugerem que, para a identificação de um professor exemplar, tanto os docentes quanto os discentes valorizaram não apenas atitudes relacionadas à docência, mas sobretudo as atitudes, o comportamento e as características pessoais do professor. Sugerem ainda a necessidade de desenvolver estratégias de valorização da pesquisa científica, inclusive com incentivos para uma maior participação tanto dos 
docentes quanto dos discentes, ressaltando sempre a sua a importância na formação médica.

\section{AGRADECIMENTOS}

Agradecemos ao Programa de Iniciação Científica (Probic) da Unifor a concessão de bolsa ao discente João Pedro Brasil Teixeira para desenvolver esta pesquisa.

\section{REFERÊNCIAS}

1. Alweshahi Y, Harley D, Cook DA. Students' perception of the characteristics of effective bedside teachers. Med. teach. 2007;29(2-3):204-9.

2. Irby DM, Papadakis M. Does good clinical teaching really make a difference? Am. j. med. 2001;110:231-2.

3. Stone S, Ellers B, Holmes D, Orgren R, Qualters D, Thompson J. Identifying oneself as a teacher: the perceptions of preceptors. Med. educ. 2002;36:180-5.

4. Sutkin G, Wagner E, Harris I, Schiffer R. What makes a good clinical teacher in Medicine? A review of the literature. Acad. med. 2008;83(5):452-66.

5. Haider SI, Snead DRJ, Bari MF. Medical students' perceptions of clinical teachers as role model. PLos ONE 2016;13(1):e0150478 [acesso em 12 junho 2019]. Disponível em: https://journals.plos.org/plosone/ article/file?id=10.1371/journal.pone.0150478\&type=printable .

6. Kiani Q, Umar S, Iqbal M. What do medical students expect in a teacher? The Clinical Teacher 2014;11:203-8.

7. Harden RM, Crosby J. AMEE Guide $n^{\circ}$ 20: The good teacher is more than a lecturer - the twelve roles of the teacher. Med. teach. 2000;22(4):334-47.

8. Ray S. Role models. BMJ 2010;340:c1572.

9. Feitosa HN, Rego S, Bataglia P, Rego G, Nunes R. Competência de juízo moral dos estudantes de medicina: um estudo piloto. Rev. bras. educ. med. 2013;37(1):5-14.

10. Vaz M. The ethics of teaching in medicine: a personal view. Indian Journal of Medical Ethics 2019;4(3):221-6.

11. D’Ignazio T, Lavoie G, Pomerani T, Lachapelle A, Gaucher N. Preexchange training - developing ethical and cultural competencies in medical students. Med. teach. 2019;41(12):1399-403.

12. Lewis, KD, Patel A, Lopreiato JO. A focus on feedback: improving learner engagement and faculty delivery of feedback in hospital medicine. Pediatr Clin N Am 2019;66:867-80.

13. Boendermaker PM, Conradi MH, Schuling J, Meyboom-de Jong B, Zwierstra RP, Metz JCM. Core characteristics of the competent general practice trainer, a Delphi study. Advances in Health Sciences Education 2003;8:111-6.

14. Mclaen M. Qualities atributes to an ideal educator by medical students: should faculty take cognizance? Med. teach. 2001;23(4):367-70.

15. Tweedie J, Hordern J, Dacre J. Advancing medical professionalism. London: Royal College of Physicians; 2018. Disponível em: https:// www.rcplondon.ac.uk/projects/outputs/advancing-medicalprofessionalism.
16. Singh SS, Dinker RP, Nirmal KS, Avneet K, Htoo Htoo KS, Ankur B. Qualities of an effective teacher: what do medical teachers think? BMC med. educ. 2013;13:128-34 [acesso em 12 jun 2019. Disponível em: https://www.ncbi.nlm.nih.gov/pmc/articles/PMC3848658/pdf/14726920-13-128.pdf.

17. Goldie J, Dowie A, Goldie A, Cotton P, Morrison J. What makes a good clinical student and teacher? An exploratory study. BMC med. educ. 2015;15:40-8 [acesso em 12 jun 2019]. Disponível em: https:// www.ncbi.nlm.nih.gov/pmc/articles/PMC4358722/.

18. Cabral, TCB. Ensino e aprendizagem de matemática na engenharia e o uso de tecnologia. CINTED-UFRGS 2005;3(2):1-14 [acesso em 12 jun 2019]. Disponível em: http://www.educadores.diaadia. pr.gov.br/arquivos/File/2010/artigos_teses/MATEMATICA/ Artigo_Cabral.pdf.

19. Rigamonti L, Dolci A, Galetta F, Stefanelli C, Hughes M, Bartsch $\mathrm{M}$, et al. Social media and e-learning use among European exercise science students. Health Promot Int. 2019;1-8.

20. Zemlo TR, Garrison HH, Partridge NC, Ley TJ. The physicianscientist: career issues and challenges at the year 2000. FASEB $\mathrm{j}$. 2000;14(2):221-30.

21. Ávila MJ, Restrepo AR. The importance of research in undergraduate medical education. Medwave 2014;14(10):e6032 [acesso em 12 jun 2019]. Disponível em: https://www.medwave.cl/link.cgi/English/ Reviews/GeneralReviews/5077.act

22. Bonilla-Velez J, Small M, Urrutia R, Lomberk G. The enduring value of research in medical education. Int J Med Students 2017;5(1):37-44.

23. Souza PMM, Nunes CA, Silveira CS, Nobrega-Therrien SM. Integração ensino-pesquisa na educação médica: perfil docente de um colegiado. Rev. bras. educ. med. 2012;36(1):14-23.

\section{CONTRIBUIÇÃO DOS AUTORES}

Lara Gurgel Fernandes Távora orientou a elaboração do projeto, execução, revisão final do artigo. Laila Teixeira Amorim, João Pedro Brasil Teixeira, Heitor Moita Mota, Thais Barroso Vieira Costa e Victor Fernandes Távora Vieira Costa realizaram a coleta, análise dos dados e escrita inicial do artigo.

\section{CONFLITO DE INTERESSES}

Os autores declaram não haver conflito de interesses neste estudo.

\section{ENDERECO PARA CORRESPONDÊNCIA}

Lara Gurgel Fernandes Távora. Avenida Engenheiro Santana Júnior, 2947, ap. 1402, Cocó, Fortaleza, CE, Brasil. CEP: 60192-205.

E-mail: laratavora@gmail.com 